\title{
On the Nonsingularity of Real Matrices
}

\author{
By A. J. Hoffman
}

\begin{abstract}
By exploiting the theory of linear inequalities, new bounds for the real eigenvalues of a real matrix are derived, along with sufficient conditions far matrix games to be completely mixed, for determinants to be positive, etc. The simple observation on which the derivation of new results and the unification of old results are based is that the typical conditions of diagonal dominance which insure the nonsingularity of matrices are essentially systems of linear inequalities on the rows of the matrices.
\end{abstract}

I. Introduction. Our purpose in this note is to derive and unify some old and new results on matrix games, and on bounds for eigenvalues, as simple consequences of the viewpoint of the theory of linear inequalities. We consider real matrices of order $n$, typically denoted by $A=\left(a_{i j}\right)$. A well-known sufficient condition for such a matrix to be nonsingular is

$$
\left|a_{i i}\right|>\sum_{j \neq i}\left|a_{i j}\right|, \quad i=0, \cdots, n-1 .
$$

If we imagine that the matrix has been multiplied suitably by a diagonal matrix, in order that the new diagonal elements be nonnegative, then we may rewrite (1.1) as

$$
a_{i i}>\sum_{j \neq i}\left|a_{i j}\right|, \quad i=0, \cdots, n-1 .
$$

An alternative way of stating (1.2) is the following: Let $M_{i}$ be the matrix with $n$ rows and $2^{n-1}$ columns, where each column has a 1 in the $i$ th row, and the other entries are +1 or -1 in all possible ways. Let the rows of $A$ be denoted by $A_{0}^{\prime}, \cdots, A_{n-1}^{\prime}$. Then (1.2) may be rewritten as

$$
A_{i}^{\prime} M_{i}>0, \quad i=0, \cdots, n-1 .
$$

Our object in this note is to characterize all possible sets of $n$ matrices $M_{0}, \cdots, M_{n-1}$, such that (1.3) implies $A$ is nonsingular (Theorem 1). As applications, we shall discover new classes of such matrices $\left\{M_{i}\right\}$, unifying material in [1], [2] and [3] on "completely mixed" games, new conditions for some matrices to have positive determinant, and new results on bounds for real eigenvalues of real matrices.

\section{Main Theorem:}

Theorem 1. Let $M_{0}, \cdots, M_{n-1}$ be $n$ matrices, each with $n$ rows, but with the number of columns of each matrix unspecified. Let $C_{i}=\left\{M_{i} x \mid x \geqq 0\right\}$. Assume that, for each $i, C_{i}$ is a pointed cone (i.e., there exists $u_{i}, i=1, \cdots, n$, such that $u_{i}^{\prime} M_{i}>0$ ). Then

\section{(1.3) implies $A$ is nonsingular}

Received August 27, 1964. This research was supported in part by the Office of Naval Research under Contract No. Nonr 3775(00), NR 047040. 
if and only if

$$
\mathrm{U}\left( \pm C_{\mathbf{i}}\right)=R^{n}
$$

Proof. Assuming (2.2), we must show that $A$ is nonsingular. The proof is essentially the same as the standard proof [4] that (1.1) implies $A$ nonsingular. If $A$ is singular, there exists a nonzero vector $y$ such that

$$
A y=0 \text {. }
$$

By (2.2), $y$ or $-y$ is contained in one of the cones $C_{i}$. Without loss of generality, assume $y$ is in some $C_{i}$. Then, from (2.3), we have $0=A_{i}{ }^{\prime} y=A_{i}{ }^{\prime} M_{i} x>0$, since each coordinate of $A_{i}{ }^{\prime} M_{i}$ is positive (by (1.3)) and $x \geqq 0, x \neq 0$.

Conversely, assume (2.2) false, i.e., there exists a vector $y$ such that, for each $i$,

$$
y \notin C_{i},
$$

and

$$
-y \notin C_{i} .
$$

From (2.4), by the hyperplane separation theorem for cones, we infer that there exists a vector $z_{i}$ such that

$$
\left(y, z_{i}\right)<0, \quad z_{i}^{\prime} M_{i} \geqq 0 .
$$

Since we know there exists $u_{i}$ satisfying $u_{i}{ }^{\prime} M_{i}>0$, we could replace $z_{i}$ in the above inequalities by $z_{i}+\alpha_{i} u_{i}$ for $\alpha_{i}$ a small positive number, and obtain, for the new $z_{i}$,

$$
\left(y, z_{i}\right)<0, \quad z_{i}^{\prime} M_{i}>0 .
$$

Similarly, from (2.5), we infer the existence of $w_{i}$ such that

$$
\left(y, w_{i}\right)>0, \quad w_{i}^{\prime} M_{i}>0 .
$$

From (2.6) and (2.7), we see that there exists a positive combination of $z_{i}$ and $w_{i}$, orthogonal to $y$. Call that vector $A_{i}$. We then have

$$
\left(y, A_{i}\right)=0, \quad A_{i}{ }^{\prime} M_{i}>0 .
$$

In this manner, we construct a matrix $A$ which is singular, yet satisfies (1.3).

III. Application. We now turn to the question of finding matrices $\left\{M_{i}\right\}$, other than those mentioned in the Introduction, for which (2.2) holds. Let us first describe a general method for discovering some classes $\left\{M_{i}\right\}$, and consider particular instances in the next section. Let $u$ be any nonzero vector in $R^{n}$, and let $L=$ $\{x \mid(x, u)=0\}$. In $L$, choose $n$ vectors $v_{0}, \cdots, v_{n-1}$ such that $x \in L$ implies $x$ is a nonnegative combination of at most $n-1$ of the vectors $\left\{v_{1}, \cdots, v_{n-1}\right\}$. (It is easy to find such a set of vectors; let $v_{1}, \cdots, v_{n-1}$ be a basis for $L$, and $\operatorname{set} v_{0}=-\sum_{i=1}^{n-1} v_{i}$.) It is clear that, if $M_{i}$ has $n$ columns consisting of $u$ and all the vectors $\left\{v_{0}, \cdots, v_{n-1}\right\}$ except $v_{i}$, then $\mathrm{U}_{C_{i}}=\{x \mid(u, x) \geqq 0\}$. In other words, $\mathrm{U}_{C_{i}}$ is a closed half-space. Therefore, $\mathrm{U}\left( \pm C_{i}\right)=R^{n}$.

As an illustration of the foregoing, we prove 
Theorem 2. Let $\beta_{0}, \beta_{1}, \cdots, \beta_{n-1}$ be nonnegative, with $\sum \beta_{i}=1$, and assume g.c.d. $\left\{j \mid \beta_{j}>0\right\}$ is relatively prime to $n$. If $A$ satisfies

$$
\begin{aligned}
& \quad \sum_{j} a_{i j}>0, i=0, \cdots, n-1, \text { and } \\
& \sum_{j} a_{i j} \beta_{-r+j}>a_{i r}, \quad i=0, \cdots, n-1, r=0, \cdots, n-1, r \neq i,
\end{aligned}
$$

or

$$
\begin{aligned}
\sum_{j} a_{i j}>0, & i=0, \cdots, n-1, \text { and } \\
\sum_{j} a_{i j} \beta_{-r+j}<a_{i r}, & i=0, \cdots, n-1, r=0, \cdots, n-1, r \neq i,
\end{aligned}
$$

$o r$

$$
\begin{aligned}
\sum_{j} a_{i j}<0, & i=0, \cdots, n-1, \text { and } \\
\sum_{j} a_{i j} \beta_{-r+j}>a_{i r}, \quad i & =0, \cdots, n-1, r=0, \cdots, n-1, r \neq i,
\end{aligned}
$$

or

$$
\begin{gathered}
\sum_{j} a_{i j}<0, i=0, \cdots, n-1, \text { and } \\
\sum_{j} a_{i j} \beta_{-r+j}<a_{i r}, \quad i=0, \cdots, n-1, r=0, \cdots, n-1, r \neq i
\end{gathered}
$$

then $|A| \neq 0$. Furthermore, the sign of $|A|$ is positive in case (a), negative in case (c), $(-1)^{n-1}$ in case (b), $(-1)^{n}$ in case $(\mathrm{d})$.

Proof. To prove the nonsingularity of $A$, we apply the discussion in the preceding paragraph. In cases (a) and (b), $u=(1, \cdots, 1)$. In cases (c) and (d), we use $-u$ in place of $u$. Let $v_{i}, i=0, \cdots, n-1$, be the vector whose $j$ th coordinate is $-\delta_{i j}+\beta_{j-i}$. One can show that any $n-1$ of the vectors $\left\{v_{i}\right\}$ are a basis of $L$, and the remaining vector is the negative of their sum. In cases (a) and (c), we use the vectors $\left\{v_{i}\right\}$. In cases (b) and (d), we use the vectors $\left\{-v_{i}\right\}$. It is then straightforward to verify the nonsingularity of $A$ in each of the four cases.

To prove our statements about the signs of $|A|$, let us first treat case (a). It is clear that, for $\lambda \geqq 0$, the matrix $A+\lambda I$ satisfies the conditions of case (a). If $\lambda$ is very large, then clearly $|A+\lambda I|>0$. By the continuity of $|A+\lambda I|$ as a function of $\lambda$, if $|A|<0$, there would have to be some positive $\lambda$, say $\lambda_{0}$, such that $\left|A+\lambda_{0} I\right|=0$, a contradiction. To treat case (d), we observe that, in that case, $-A$ satisfies the conditions of (a). Therefore, $|A|=(-1)^{n}|-A|$ has the sign $(-1)^{n}$.

In case (b), denote by $J$ the matrix every entry of which is unity. Then $A+\lambda(J-I)$, for $\lambda \geqq 0$, satisfies the conditions of (b). But, for $\lambda$ large and positive, $|A+\lambda(J-I)|$ has the same sign as determinant $|J-I|$, which has the sign $(-1)^{n-1}$. As before, the proof is completed by appealing to the continuity of $|A+\lambda(J-I)|$. Finally, case (c) is handled by observing that, in that instance, $-A$ is covered by (b).

Theorem 3. Let A satisfy any of the four conditions stated in Theorem 2 . Then the 
system of equations $\sum_{i} a_{i j} x_{i}=1$ has a (unique) solution in which each $x_{i}>0$ in cases (a) and (b), each $x_{i}<0$ in cases (c) and (d).

l'roof. We shall only treat case (a), the proofs of the other cases being similar. By virtue of what has gone before, all we need prove is that the determinant of the matrix obtained by replacing the $i$ th row of $A$ by the vector $u$ is not zero, and has the same sign as $|A|$. Observe that the new matrix satisfies the conditions of case (a), except that the strong inequalities are replaced by weak inequalities. Since the determinant of a matrix is a continuous function of its entries, it follows that the determinant, if not zero, has the same sign as the determinant of $A$. Suppose $y$ is a vector annihilated by the new matrix. Then, since $y$ is orthogonal to $u, y \in L$. Therefore, $y$ is a nonnegative combination of at most $n-1$ of the vectors $\left\{v_{i}\right\}$. Suppose that the vector omitted in this nonnegative combination is $v_{k}, k \neq i$. Then, since $A_{k}{ }^{\prime}$ makes a positive inner product with each vector in $\left\{v_{i}\right\}$ other than $v_{k}, A_{k}{ }^{\prime}$ could not be orthogonal to $y$. If the vector omitted is $v_{i}$, replace $y$ by $-y$. It cannot happen that, also for $-y$, the vector omitted is again $v_{i}$, for it is easy to show that the cone in $L$ formed by any $n-1$ of the $v$ 's is pointed. Thus, the new matrix cannot be singular, and our theorem is proven.

In the case when $\beta_{n-1}=1$ (and all other $\beta_{i}$ are zero), Theorems 1 and 2 include the results of [3]. In the case when all $\beta_{i}$ are the same (i.e., $1 / n$ ) our theorem gives another easy sufficient condition for a matrix game to be "completely mixed." These results dispose of the "sticky" example cited in [1] and [2].

IV. Bounds for Real Eigenvalues of Real Matrices. It is well known [4] that, from conditions on the coefficients of a matrix which insure nonsingularity, one may derive bounds for the eigenvalues of that matrix. In order to apply the foregoing results, it is useful for us to first state an alternative form of Theorem 2.

Theorem $2^{\prime}$. Let $\beta_{0}, \beta_{1}, \cdots, \beta_{n-1}$ satisfy the conditions of Theorem 2 . Consider any subset $S \subset\{0,1, \cdots, n-1\}$, and let $\bar{S}$ be the complementary set of indices. If inequalities (a) of Theorem 2 are satisfied for $i \in S$, and inequalities (d) are satisfied for $i \in \bar{S}$, then $A$ is nonsingular. Similarly, if inequalities (b) are satisfied for $i \in S$, and inequalities (c) are satisfied for $i \in \bar{S}$, then $A$ is nonsingular.

Proof. Going back to Theorem 1, the only change we have made in Theorem 2 is to replace $C_{i}$, for $i \in \bar{S}$, by $-C_{i}$. By virtue of $(2.2)$, this change does not affect the nonsingularity of $A$.

It is simplest for us to consider the case of Theorem 2 in which all $\beta_{i}$ are $1 / n$. (Conditions somewhat more complicated to state are inferrable when the $\beta_{i}$ are any nonzero numbers, not necessarily the same.) Then the inequalities in each of (a), (b), (c), (d) become, respectively,

$$
\begin{aligned}
& \sum_{j} a_{i j}>0, \\
& \sum_{j} a_{i j}>n \max _{j \neq i} a_{i j}, \\
& \sum_{j} a_{i j}>0 \\
& \sum_{j} a_{i j}<n \min _{j \neq i} a_{i j},
\end{aligned}
$$


(c)

$$
\begin{aligned}
& \sum_{j} a_{i j}<0, \\
& \sum_{j} a_{i j}>n \max _{j \neq i} a_{i j}, \\
& \sum_{j} a_{i j}<0, \\
& \sum_{j} a_{i j}<n \min _{j \neq i} a_{i j} .
\end{aligned}
$$

We shall also use, for any real number $a$, the symbols $a_{+}$and $a_{-}$, where

$$
\begin{aligned}
a_{+}=a & \text { if } a \geqq 0, \\
=0 & \text { if } a<0, \\
a_{-}=a & \text { if } a \leqq 0, \\
=0 & \text { if } a>0 .
\end{aligned}
$$

Theorem 4. For any real matrix $A$ define

$$
\begin{aligned}
& P_{i}=\sum_{j} a_{i j}-\left(n \max _{j \neq i} a_{i j}\right)_{+}, \\
& Q_{i}=\sum_{j} a_{i j}-\left(n \min _{j \neq i} a_{i j}\right)_{-} .
\end{aligned}
$$

Then every real eigenvalue $\lambda$ of $A$ lies in the union of the closed intervals $\left[P_{i}, Q_{i}\right]$.

Furthermore, no eigenvalue of $A$ satisfies the inequalities

$$
\text { (4.1). } \max _{i}\left\{\sum_{j} a_{i j}-\left(n \min _{j \not i} a_{i j}\right)+\right\}<\lambda<\min _{i}\left\{\sum_{j} a_{i j}-\left(n \max _{j \neq i} a_{i j}\right)-\right\} \text {. }
$$

Proof. To prove the first part of the theorem, assume that the real eigenvalue $\lambda$ of $A$ does not lie in any of the intervals $\left[P_{i}, Q_{i}\right]$. Since the $i$ th interval contains $\sum_{j} a_{i j}$, it follows that, for each $i, \lambda \neq \sum_{j} a_{i j}$. Let $S=\left\{i \mid \sum_{j} a_{i j}-\lambda>0\right\}$, $\bar{S}=\left\{i \mid \sum_{j} a_{i j}-\lambda<0\right\}$. Then, if $i \in S$, the $i$ th row of $A-\lambda I$ satisfies inequality (a). If $i \in \bar{S}$, the $i$ th row of $A-\lambda I$ satisfies inequality (d). By virtue of Theorem $2^{\prime}, A-\lambda I$ is nonsingular, contradicting the fact that $\lambda$ is an eigenvalue of $A$.

To prove that no eigenvalue of $A$ satisfies (4.1), let us first show that, if $\lambda$ satisfies (4.1), then, for each $i, \lambda \neq \sum_{j} a_{i j}$. If not, then for some $i$, we have

$$
\sum_{j} a_{i j}-\left(n \min _{j \neq i} a_{i j}\right)_{+}<\sum_{j} a_{i j}<\sum_{j} a_{i j}-\left(n \max _{j \neq i} a_{i j}\right)-.
$$

If $\sum_{j} a_{i j}$ is subtracted from each expression, the resulting inequalities are inconsistent. Now, with $S$ and $\bar{S}$ defined as before, we find that each row of $A-\lambda I$ satisfies either (b) or (c), and the argument is the same as before. In the case of the matrix $a I+b J$, where $J$ is the matrix every entry of which is unity, this theorem establishes that the only possible real eigenvalues of $A$ are $a+n b$ and $a$, which is indeed the case. 
1. H. F. Bohnenblust, S. Karlin \& L. S. Shapley, "Solutions of discrete, two-person games," Contributions to the Theory of Games, Annals of Mathematics Studies No. 24, Princeton Univ. Press, Princeton, N. J., 1950, p. 51-72. MR 12, 513.

2. A. J. Goldman, "Recognition of completely mixed games," J. Res. Nat. Bur. Standards Sect. $B$, v. $67 \mathrm{~B}, 1963$, p. $23-29$.

3. L. Negrescu, A. Németh \& T. Rus, "Sur les solutions positives d'un systeme d'équations linéaires," Mathematica (Cluj), v. 4 (27), 1962, p. 65-69.

4. Olga TAUSSKY, "A recurring theorem on determinants," Amer. Math. Monthly, v. 56, 1949, p. 672-676. MR 11, 307. 